\title{
Celebrity doctor encourages kids to brush their teeth
}

NHS doctor, TV presenter, author and celebrity contestant on 'Strictly Come Dancing' Dr Ranj is the star of three new mini-videos aimed at giving parents, carers and children the tips they need to keep teeth healthy. Actor Gemma Oaten who rose to fame in TV soap 'Emmerdale' supports Dr Ranj as the voice of sidekick Supertooth.

The videos combine live action with computer animation. Dr Ranj and Supertooth guide children in different age categories and demonstrate, in just a few minutes, how to brush teeth.

The videos were instigated for the British Society of Paediatric Dentistry (BSPD) by real life Toothfairy Claire Stevens, a Consultant in Paediatric Dentistry and a spokesperson for BSPD. She explained: 'Every year, when statistics for dental extractions in children are published, I am interviewed by the media who are horrified that so many children are ending up in hospital having teeth taken out and they want to know why.

'As a children's dentist, I know that the main culprit is frequent consumption of sugary foods and drinks, especially between meals. We also know that it can be a challenge to teach children to brush their teeth when they are young and then encourage them to keep brushing as they get older. We had to find a way to reach parents with helpful advice in a fun and nonjudgemental way'

Coincidentally, Claire was contacted by Ben Underwood, a dentist and the creator of the free Brush DJ app, who was looking for videos that would give parents and carers accurate information in the most accessible

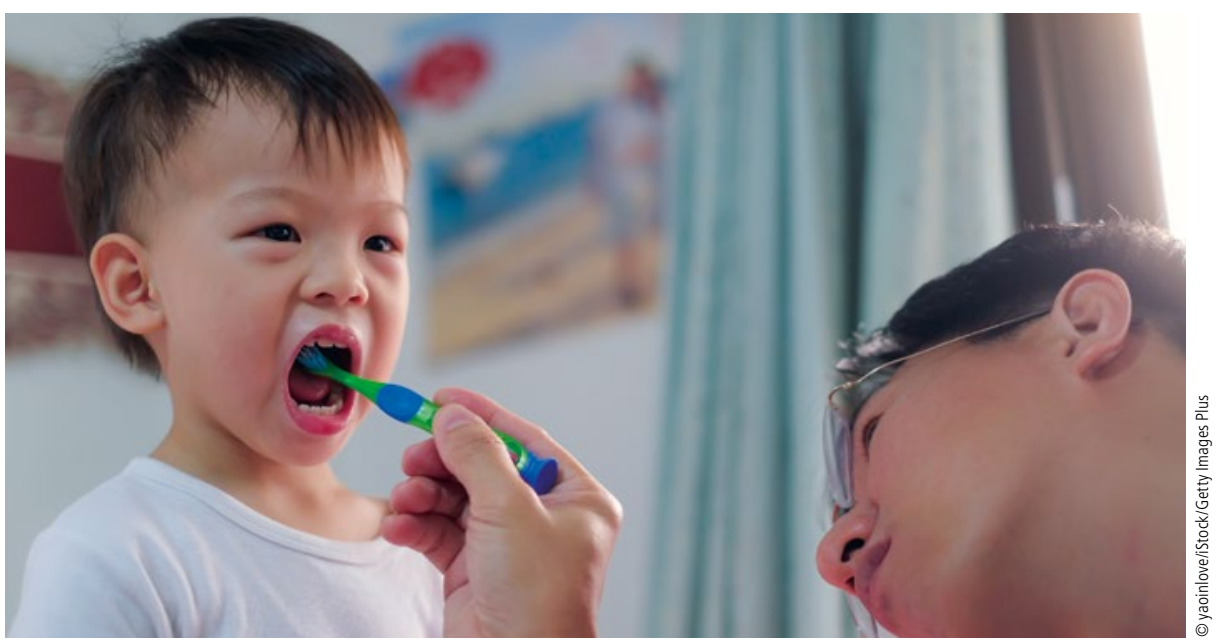

format - short, fun and online.

Claire went on a mission to speak to Dr Ranj who had co-created and presented the CBeebies show Get Well Soon. Her daughter loved the programme and would quote $\mathrm{Dr}$ Ranj after watching the show.

'As soon as I spoke to him, I knew he was the right person to help make some watchable videos for children. He was immediately on board with the messages and he insisted on giving of his time freely.'

Now in collaboration, Ben and Claire carried out research among high-risk patients supported by BSPD Exec member, Hannah Walsh. They learned that the parents of the target group favoured videos which are short, fun, have star quality and include animation. All aspects of the videos, the length, format, design and distribution, have been tailored in response to the feedback they received. The results are now being written up by Dr Walsh as an academic paper.

The final crucial pieces in the jigsaw, said
Claire, were production and sponsorship. They were fortunate to have Joff Powell and his team at Pedwar Productions make the videos.

Dr Ranj said: 'Every child deserves to grow up happy and healthy, and that absolutely includes having healthy teeth. I've seen so many instances where dental health is forgotten, or people just don't realise its importance, until it's too late.

'The statistics around how many children have to have teeth removed because of decay are shocking. All it takes to prevent this situation is some simple advice and practice, which is why I'm so keen to be part of this campaign.

The links to view the videos are as follows:

0-3 video: https://www.youtube.com/ watch? $=$ owbp5F0K $45 \mathrm{c} \& \mathrm{t}=7 \mathrm{~s}$

3-6 video: https://www.youtube.com/ watch?v=lQE4xxk1r5g

7+ video: https://www.youtube.com/ watch? $\mathrm{v}=$ GHS27DHyIi0.

\section{Female sugary drink consumption linked to CVDs}

Drinking one or more sugary beverages a day was associated with a nearly $20 \%$ greater likelihood of women having a cardiovascular disease compared to women who rarely or never drank sugary beverages, according to new research ${ }^{1}$ published in the Journal of the American Heart Association, an open access journal of the American Heart Association.

In the large, ongoing California Teachers Study, which began in 1995, drinking one or more of any type of sugary beverage daily was associated with a $26 \%$ higher likelihood of needing a revascularisation procedure, such as angioplasty to open clogged arteries, and a $21 \%$ higher likelihood of having a stroke compared to women who rarely or never drank sugary beverages. Sugary beverages in this study were defined as caloric soft drinks, sweetened bottled waters or teas and sugar-added fruit drinks, not $100 \%$ fruit juices.
The study included more than 106,000 women, with an average age of 52, who had not been diagnosed with heart disease, stroke or diabetes when they enrolled in the study.

\section{Reference}

1. Pacheco L S, Lacey Jr J V, Martinez M E et al. Sugar-sweetened beverage intake and cardiovascular disease risk in the California Teachers Study. J Am Heart Assoc 2020; 9: e014883. doi: 10.1161/ JAHA.119.014883. 